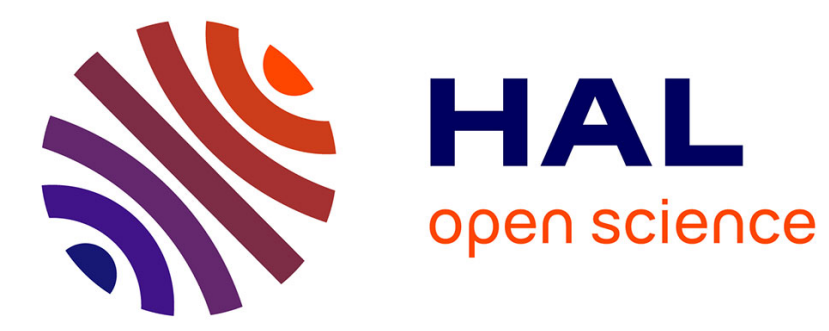

\title{
Homoclinic orbits to invariant sets of quasi-integrable exact maps
}

Patrick Bernard

\section{To cite this version:}

Patrick Bernard. Homoclinic orbits to invariant sets of quasi-integrable exact maps. Ergodic Theory and Dynamical Systems, 2000, 20 (6), pp.1583-1601. hal-01251209

\section{HAL Id: hal-01251209 \\ https://hal.science/hal-01251209}

Submitted on 5 Jan 2016

HAL is a multi-disciplinary open access archive for the deposit and dissemination of scientific research documents, whether they are published or not. The documents may come from teaching and research institutions in France or abroad, or from public or private research centers.
L'archive ouverte pluridisciplinaire HAL, est destinée au dépôt et à la diffusion de documents scientifiques de niveau recherche, publiés ou non, émanant des établissements d'enseignement et de recherche français ou étrangers, des laboratoires publics ou privés. 


\title{
Homoclinic orbits to invariant sets of quasi-integrable exact maps
}

\author{
Patrick Bernard \\ october 98
}

In Hamiltonian mechanics, various questions and results concern the becoming of the invariant tori of an integrable system after perturbation. It is well known, by KAM theory, that many of these invariant tori are deformed but not destroyed. But it is known as well that many of them are destroyed. They give rise to more or less complicated invariant sets such as Mather invariant sets [9].

One particular kind of tori that can be destroyed is resonant tori, that is invariant tori foliated by lower dimensional invariant tori. Treschev proved in [11] that if the frequency induced on the lower dimensional torus satisfies some Diophantine condition, then one of the lower dimensional tori is preserved, and becomes hyperbolic (whiskered in Arnold's terminology [1]). If the unperturbed Hamiltonian is positive definite, it has been proved by Bolotin [3] that this preserved torus admits homoclinic orbits in the perturbed system. Similar results have been obtained by Eliasson [5] for 1-resonant tori without the convexity assumption. These results are important not only as generalizations to high dimensional systems of old results on twist maps, but also because they are a step in the attempt to generalize Arnold's construction of [1] and study diffusion in high dimensional Hamiltonian systems. Unfortunately, as is explained in [8], there are not enough preserved tori for Arnold's construction to be led directly. That's why the case of resonant tori which do not satisfy Diophantine conditions and which can be completely destroyed is of some interest. Bolotin gives partial answer to that question in [3], obtaining semi-asymptotic orbits to the Mather invariant set but does not obtain an invariant set with homoclinic orbits.

In this paper, we focus our attention to a general resonant torus no lower dimensional torus of which has to be preserved. We prove that its destruction gives rise to compact invariant sets, the Peierl's sets introduced by Mather in [10] and containing the usual Mather sets, and that these sets admit nontrivial homoclinic orbits. As we stressed above, this result could have some interest in the study of diffusion, allowing to fill the gaps between preserved tori. Our method, that provides a different and less involved proof of Bolotin's result, is based on periodic orbits: both the invariant sets and the homoclinic orbits are obtained as limits of periodic orbits which are found by the variational methods of [2]. That the Peierl's set is the natural asymptotic set to be considered has just been noticed by Fathi [6] by quite different methods.

For convenience, we will consider exact maps instead of Hamiltonian flows, the links between both theories are well known. Let us quote for instance [7] or [2] for details about convexity assumptions. We also refer to [2] where it is explained how the local study of any quasi-integrable exact map around a positive definite torus can be reduced to the following setting. 


\section{Main Result}

We will consider an integrable symplectic diffeomorphism

$$
\Phi_{0}: \mathbb{T}^{n} \times \mathbb{R}^{n} \longrightarrow \mathbb{T}^{n} \times \mathbb{R}^{n}
$$

whose pull back

$$
\phi_{0}: \mathbb{R}^{n} \times \mathbb{R}^{n} \longrightarrow \mathbb{R}^{n} \times \mathbb{R}^{n}
$$

can be written

$$
\phi_{0}(x, y)=(x+\omega(y), y) .
$$

In all the following, we will suppose that $\Phi_{0}$ satisfies the following two hypothesis:

Hypothesis 1 (non-degeneracy) the map $\omega: \mathbb{R}^{n} \longrightarrow \mathbb{R}^{n}$ is a diffeomorphism.

Then $\phi_{0}$ admits a global generating function of type $S$ [4], i.e. there exists a function $S$ : $\mathbb{R}^{n} \times \mathbb{R}^{n} \longrightarrow \mathbb{R}$ such that

$$
\left(x_{1}, y_{1}\right)=\phi_{0}\left(x_{0}, y_{0}\right) \Longleftrightarrow\left\{\begin{array}{l}
y_{0}=\partial_{1} S\left(x_{0}, x_{1}\right) \\
y_{1}=-\partial_{2} S\left(x_{0}, x_{1}\right)
\end{array} .\right.
$$

It is easy to see that in our case

$$
S\left(x_{0}, x_{1}\right)=h\left(x_{1}-x_{0}\right),
$$

and we will make the following second assumption

Hypothesis 2 (convexity) the function $h: \mathbb{R}^{n} \longrightarrow \mathbb{R}$ is strictly convex.

Let $\Phi$ be a $C^{1}$ exact perturbation of $\Phi_{0}$, its pull back $\phi$ has a $C^{2}$ generating function

$$
S\left(x_{0}, x_{1}\right)=h\left(x_{1}-x_{0}\right)+\epsilon P\left(x_{0}, x_{1}\right),
$$

where the perturbation $P$ satisfies

Hypothesis 3 (exactness) $P\left(x_{0}+m, x_{1}+m\right)=P\left(x_{0}, x_{1}\right)$ for all $m \in \mathbb{Z}^{n}$.

Since we are interested in the dynamical structure of a bounded region of phase space, we can change the mapping at infinity and furthermore suppose (see [2])

Hypothesis 4 (localization) there is a compact set $K \subset \mathbb{R}^{n}$ and a number $a>0$ such that

$$
S(x, x+t)=h(t)+\epsilon P(x, x+t)=a|t|^{2}+0
$$

when $t \notin K$.

Let $y_{0}$ and $\omega_{0}=\omega\left(y_{0}\right)$ be fixed. Assume that $\omega_{0}$ is resonant, which means that there exists $k \in \mathbb{Z}^{n}$ such that $<k, \omega_{0}>\in \mathbb{Z}$. Let

$$
R=\left\{k \in \mathbb{Z}^{n} \text { such that }<k, \omega_{0}>\in \mathbb{Z}\right\}
$$

and let $r=\operatorname{rank}(R)$, then we say that $\omega_{0}$ is $r$-resonant. The free group $R$ has a basis $B$ of cardinal $r$. It is not hard to see that there is a foliation of the torus $y=y_{0}$ in ergodic subtori and a group morphism

$$
F: \mathbb{T}^{n} \times \mathbb{T}^{n-r} \longrightarrow \mathbb{T}^{n}
$$


such that these tori are

$$
\left\{F(x, \theta), \theta \in \mathbb{T}^{n-r}\right\} .
$$

The unperturbed map restricted to $\left\{y=y_{0}\right\}$ is given by

$$
F(x, \theta) \longmapsto F(x, \theta)+\omega_{0}=F\left(x, \theta+\tilde{\omega}_{0}\right) .
$$

The frequency $\tilde{\omega}_{0} \in \mathbb{R}^{n-r}$ is called the induced frequency. It is useful to define the averaged perturbation

$$
\mathcal{P}: \mathbb{T}^{n} \longrightarrow \mathbb{R}
$$

given by

$$
\begin{aligned}
\mathcal{P}(x) & =\int_{\mathbb{T}^{n-r}} P\left(F(x, \theta), F(x, \theta)+\omega_{0}\right) \mathrm{d} \theta \\
& =\lim _{N \rightarrow+\infty} \frac{1}{2 N} \sum_{i=-N}^{N} P\left(x+i \omega_{0}, x+(i+1) \omega_{0}\right) .
\end{aligned}
$$

The function $\mathcal{P}$ is then constant along the ergodic subtori,

$$
\mathcal{P}(F(x, \theta))=\mathcal{P}(x) .
$$

Hypothesis $5 \mathcal{P}$ is minimal on exactly one of the ergodic tori $\left\{F(x, \theta), \theta \in \mathbb{T}^{n-r}\right\}$, which is a non-degenerate critical manifold for $\mathcal{P}$. We will note $\Gamma_{0}$ this minimal torus.

We will use the notation $\pi$ both for the canonical projection $\mathbb{R}^{n} \longrightarrow \mathbb{T}^{n}$ and for $(\pi, \mathrm{Id})$ : $\mathbb{R}^{n} \times \mathbb{R}^{n} \longrightarrow \mathbb{T}^{n} \times \mathbb{R}^{n}$ and $\Pi$ for the first factor projection $\mathbb{T}^{n} \times \mathbb{R}^{n} \longrightarrow \mathbb{T}^{n}$ or $\mathbb{R}^{n} \times \mathbb{R}^{n} \longrightarrow \mathbb{R}^{n}$. We also set

$$
U_{\delta}=\left\{q \in \mathbb{T}^{n}, \operatorname{dist}\left(q, \Gamma_{0}\right)<\delta\right\},
$$

and we will write $U_{\delta}$ for $\pi^{-1}\left(U_{\delta}\right)$. We are now in a position to state our main result:

Theorem 1 Let $\Phi_{\epsilon}$ be the exact map whose pullback $\phi_{\epsilon}$ is generated by a $C^{2}$ function

$$
S_{\epsilon}\left(x_{0}, x_{1}\right)=h\left(x_{1}-x_{0}\right)-\epsilon P\left(x_{0}, x_{1}\right)
$$

satisfying $\boldsymbol{H}$ 1-5. Let $C>0$ be any constant, then there are two nonnegative constants $\Delta$ and $A$ and a function $\delta(\epsilon)$ with $\lim _{\epsilon \rightarrow 0} \delta=0$ such that for any $\epsilon \leqslant \Delta$ and any $c \in \mathbb{R}^{n}$ with $\left\|c-\nabla h\left(\omega_{0}\right)\right\| \leqslant C \epsilon$, there exists a compact invariant set $\tilde{\Sigma}_{c}(\epsilon)$ of $\Phi_{\epsilon}$ satisfying

- $\Pi_{\mid \tilde{\Sigma}_{c}(\epsilon)}$ is a bilipschitz homeomorphism onto its image.

- $\tilde{\Sigma}_{c}(\epsilon) \subset\left\{(x, y) \in \mathbb{T}^{n} \times \mathbb{R}^{n} / x \in U_{\delta(\epsilon)},\left\|y-y_{0}\right\| \leqslant A \sqrt{\epsilon}\right\}$,

- $\tilde{\Sigma}_{c}(\epsilon)$ has $r+1$ homoclinic orbits lying in the zone $\left\{\left\|y-y_{0}\right\| \leqslant A \sqrt{\epsilon}\right\}$ of phase space.

- Both the invariant sets and the homoclinic orbits belong to $\overline{\operatorname{Per}(\Phi)}$.

The invariant set $\tilde{\Sigma}_{c}(\epsilon)$ is a Peierl's set as defined in Mather [10], it will be described with some details in section 3. If the induced frequency $\tilde{\omega}_{0}$ satisfies some Diophantine condition, and if the maps involved are sufficiently regular, Treschev [11] obtains the existence of an hyperbolic invariant torus of frequency $\omega_{0}$. In this case, theorem 1 becomes: 
Theorem 2 (Bolotin,[3]) There is a constant $C>0$ such that if there exists a KAM hyperbolic torus $\Gamma$ with rotation vector $\omega_{0}$, then there is $c$ satisfying the hypothesis of theorem 1 such that

$$
\tilde{\Sigma}_{c}(\epsilon)=\Gamma .
$$

The torus $\Gamma$ thus admits $r+1$ homoclinics.

REMARK : The existence of $2 r$ homoclinic orbits may actually be proved in both statements above by noticing that the system is almost reversible in the region of phase space under interest.

In Section 2, we introduce the variational setting for periodic orbits that will be used in all the following. In section 3, we describe the asymptotic set. We first state some general properties of Peierl's sets, proving results of [10] in order to stress that no minimal measures are needed to introduce the Peierl's set. In the subsections we use averaging methods inspired from [3] to localize the asymptotic set. In section 4, we explain that certain sequences of periodic orbits converge to homoclinic orbits, and in section 5 , we use this fact to get several nontrivial homoclinics.

\section{Periodic orbits}

All the constructions below will be based on periodic orbits, that will be obtained as action minimizers. We will first introduce a variational problem for orbits of $\phi$ in the universal cover $\mathbb{R}^{n} \times \mathbb{R}^{n}$. Let

$$
E_{w}^{T}=\left\{\left(x_{0}, \ldots, x_{T}\right) \in\left(\mathbb{R}^{n}\right)^{T+1} \text { such that } x_{T}=x_{0}+w\right\},
$$

Consider the Lagrangian

$$
\begin{gathered}
L_{w}^{T}: E_{w}^{T} \longrightarrow \mathbb{R} \\
L_{w}^{T}\left(x_{0}, \ldots, x_{T}\right)=\sum_{i=0}^{T-1} S\left(x_{i}, x_{i+1}\right) .
\end{gathered}
$$

It is not hard to see that there exists a $T$-periodic orbit

$$
\left(\left(x_{0}, y_{0}\right),\left(x_{1}, y_{1}\right), \ldots,\left(x_{T}, y_{T}\right)\right)
$$

of $\phi$ if and only if

$$
\left(x_{0}, \ldots, x_{T}\right) \in \operatorname{crit}\left(L_{w}^{T}\right) .
$$

The orbit having a given $x$-projection is then uniquely defined, it satisfies:

$$
y_{i}=\partial_{1} S\left(x_{i}, x_{i+1}\right)=-\partial_{2} S\left(x_{i-1}, x_{i}\right) .
$$

This variational setting has been introduced and studied in [2], we will need the following part of their result, in which only estimate (2) is non-trivial.

Theorem 3 (Bernstein Katok) Given $C>0$, there exist two non negative constants $\Delta$ and $A$, depending on $h$ and $C$ but neither on $w$ or $T$ nor on $P$, such that if $\|d P\|_{C^{0}} \leqslant C$ and $\epsilon \leqslant \Delta$, the above variational problem $L_{w}^{T}$ admits a minimizing orbit $X=\left(x_{i}\right)$ satisfying

$$
\left|x_{i+1}-x_{i}-w / T\right| \leqslant A \sqrt{\epsilon} .
$$


There are corresponding problems on $\mathbb{T}^{n}$. For any $c \in \mathbb{R}^{n}$, let

$$
S_{c}\left(x_{0}, x_{1}\right)=S\left(x_{0}, x_{1}\right)-<c, x_{1}-x_{0}>.
$$

The functional ${ }_{c} L_{w}^{T}$ associated to $S_{c}$ has the same critical points and the same minima than that associated to $S$. We can now define

$$
E^{T}=\left\{\left(q_{0}, \ldots, q_{T}\right) \in\left(\mathbb{T}^{n}\right)^{T+1} \text { such that } q_{T}=q_{0}\right\}
$$

and

$$
H_{c}^{T}\left(q_{0}, \ldots, q_{T}\right)=\sum_{i=0}^{T-1} H_{c}\left(q_{i}, q_{i+1}\right)
$$

where

$$
H_{c}\left(q_{0}, q_{1}\right)=\min _{\pi\left(x_{0}\right)=q_{0}, \pi\left(x_{1}\right)=q_{1}}\left(S_{c}\left(x_{0}, x_{1}\right)\right)
$$

In the following, we will usually use $q$ for points and orbits in $\mathbb{T}^{n}$ and $x$ for points and orbits in $\mathbb{R}^{n}$. Given $T$ and $c$, it is easy to see that the function

$$
w \in \mathbb{Z}^{n} \longmapsto \min \left({ }_{c} L_{w}^{T}\right)
$$

is proper. It must have a minimum, and we will call $W_{c}^{T}$ the set of rotation numbers $w \in \mathbb{Z}^{n}$ realizing this minimum. If $X$ is a minimizing orbit of $L_{w}^{T}$ with $w \in W_{c}^{T}$ then it is easy to see that its projection $Q \in E^{T}$ is a minimizer of $H_{c}^{T}$, and any minimizer of $H_{c}^{T}$ can be obtained that way. The minimizing orbits of the functionals $H_{c}$ depend on $c$, and if $w, T$ and $c$ are fixed, the projection $Q \in E^{T}$ of a minimizing orbit $X \in E_{w}^{T}$ of $L_{w}^{T}$ need not be a minimizing orbit of $H_{c}^{T}$. Following Mather, we will call configurations the elements of $E_{w}^{T}$ or $E^{T}$ or in general all sequences of points. Let us end this section with an important remark:

Lemma 1 The function

$$
H_{c}: \mathbb{T}^{n} \times \mathbb{T}^{n} \longrightarrow \mathbb{R}
$$

is Lipschitz continuous.

Proof: Let us first notice that there is a constant $C$ depending on $c$ such that for any $(q, p) \in$ $\left(\mathbb{T}^{n}\right)^{2}$ there are covering points $(x, y)$ of $(q, p)$ with $\|(x, y)\| \leqslant C$ and $H_{c}(q, p)=S_{c}(x, y)$. Let us now take two other points $q^{\prime}$ and $p^{\prime}$ and their covering points $x^{\prime}$ and $y^{\prime}$ closest to $x$ and $y$. The result follows from the inequality

$$
H_{c}\left(q^{\prime}, p^{\prime}\right)-H_{c}(q, p) \leqslant S_{c}\left(x^{\prime}, y^{\prime}\right)-S_{c}(x, y) \leqslant K\left\|\left(x^{\prime}, y^{\prime}\right)-(x, y)\right\| \leqslant K d\left(\left(q^{\prime}, p^{\prime}\right),(q, p)\right) .
$$

\section{$3 \quad$ The asymptotic set}

We will now define and describe the invariant set to which homoclinic orbits will be searched. Let

$$
\alpha(c)=\inf _{T \geqslant 0}\left(\frac{\min H_{c}^{T}}{T}\right) .
$$

This function is the $\alpha$ function of Mather. The following lemma will be of great importance: 


\section{Lemma 2}

$$
\liminf _{T \longrightarrow \infty}\left(\min \left(H_{c}^{T}-T \alpha(c)\right)\right)=0 .
$$

Proof: Let us set $m_{T}=\min \left(H_{c}^{T}-T \alpha(c)\right)$. If for some $T$ we have $m_{T}=0$ then calling $Q_{T}$ the minimizing sequence of $H_{c}^{T}-T \alpha(c)$ and $n * Q_{T}$ the $n T$-periodic configuration consisting of $n$ iterates of $Q_{T}$ we have $H_{c}^{n T}\left(n * Q_{T}\right)-n T \alpha(c)=0$ for any $n$, and so $\lim \inf m_{T}=0$. Thus if we assume that $\lim \inf m_{T}>0$, then there is a real number $l$ satisfying

$$
m_{T} \geqslant l>0 \text {. }
$$

Let us fix $r>0$ such that $\left|H_{c}(z, x)-H_{c}(z, y)\right| \leqslant l / 2$ when $d(x, y) \leqslant 2 r$. There is a real number $p$ with $0<p<1$ such that, given $T$ points on the torus, there is a ball of radius $r$ containing at least $p T$ of them. Take a minimizing configuration $Q^{T}=\left(q_{i}^{T}\right)$ of $H_{c}^{T}$, after permutation of the indices, there are $K \geqslant p T$ points $q_{0}^{T}, q_{i_{1}}^{T}, \ldots, q_{i_{K-1}}^{T}$ contained in a ball of radius $r$. The configurations $Q_{k}=\left(q_{i_{k-1}}^{T}, q_{i_{k-1}+1}^{T}, \ldots, q_{i_{k}}^{T}\right)$ of length $T_{k}=i_{k}-i_{k-1}$ are almost periodic and we have

$$
H_{c}^{T_{k}}\left(Q_{k}\right)-T_{k} \alpha(c) \geqslant l / 2,
$$

adding these inequalities for all $k$ we get

$$
H_{c}^{T}(Q)-T \alpha(c) \geqslant K l / 2 \geqslant p T l / 2 .
$$

This gives

$$
\alpha(c) \leqslant H_{c}^{T} / T-p l / 2
$$

which is in contradiction with the above definition of $\alpha$.

The following definition is that of [10], but we avoid the use of minimal measures.

Definition 1 A Peierl's point is a point $q \in \mathbb{T}^{n}$ such that there exists a sequence $Q^{n}$ of $T_{n}$-periodic configurations with $q_{0}^{n}=q$ and

$$
\lim _{n \rightarrow \infty} H_{c}^{T_{n}}\left(Q^{n}\right)-T_{n} \alpha(c)=0 .
$$

The set of all Peierl's points is the Peierl's set $\Sigma_{c}$.

That this set is not empty is an easy consequence of lemma 2. Take a sequence $Q_{n}$ of $T_{n^{-}}$ periodic configurations such that $\lim _{n \longrightarrow \infty} H_{c}^{T_{n}}\left(Q^{n}\right)-T_{n} \alpha(c)=0$. Any accumulation point of the bounded sequence $\left(q_{0}^{n}\right)_{n \in \mathbb{N}}$ is a Peierl's point. In order to get further information on the set $\Sigma_{c}$, we will need the

Lemma 3 Let us fix $q_{0} \in \mathbb{T}^{n}$ and let $Q^{n}$ and $P^{n}$ be two periodic configurations with $q_{0}^{n}=$ $q_{0}=p_{0}^{n}$ and

$$
\lim _{n \longrightarrow \infty}\left(H_{c}-T \alpha\right)\left(Q^{n}\right)=0=\lim _{n \longrightarrow \infty}\left(H_{c}-T \alpha\right)\left(P^{n}\right),
$$

then $q_{1}^{n}$ and $p_{1}^{n}$ have the same limit $q_{1}$.

Proof: We first take subsequences so that $q_{1}^{n}, p_{1}^{n}, q_{T_{q}^{n}-1}^{n}$ and $p_{T_{p}^{n}-1}^{n}$ have limits $q_{1}, p_{1}, q_{-1}$ and $p_{-1}$. Consider the configurations

$$
R^{n}=\left(q_{0}^{n}, q_{1}^{n}, \ldots, q_{T_{q}^{n}-1}^{n}, q, p_{1}^{n}, \ldots, p_{T_{p}^{n}}^{n}\right)
$$


and

$$
R^{n}(q)=\left(q_{0}^{n}, q_{1}^{n}, \ldots, q_{T_{q}^{n}-1}^{n}, q_{0}, p_{1}^{n}, \ldots, p_{T_{p}^{n}}^{n}\right), q \in \mathbb{T}^{n}
$$

and let

$$
h^{n}(q)=H_{c}\left(R^{n}(q)\right)-T_{n} \alpha(c),
$$

where $T_{n}=T_{p}^{n}+T_{q}^{n}$. We then have

$$
h^{n}(q)=c_{n}+H_{c}\left(q_{T_{q}^{n}-1}^{n}, q\right)+H_{c}\left(q, p_{1}^{n}\right) .
$$

Since $h^{n}\left(q_{0}\right) \longrightarrow 0$ the sequence $c_{n}$ has a limit $-H_{c}\left(q_{-1}, q_{0}\right)-H_{c}\left(q_{0}, p_{1}\right)$. The function $h^{n}(q)$ thus has a limit $h(q)$ which satisfies $h\left(q_{0}\right)=0$ and $h \geqslant 0$. It follows that the function

$$
q \longmapsto H_{c}\left(q_{-1}, q\right)+H_{c}\left(q, p_{1}\right)
$$

must have a minimum at $q_{0}$. Taking points $x_{-1}, x_{0}$ and $y_{1}$ in $\mathbb{R}^{n}$ covering $q_{-1}, q_{0}$ and $p_{1}$ and such that $H_{c}\left(q_{-1}, q_{0}\right)=S_{c}\left(x_{-1}, x_{0}\right)$ and $H_{c}\left(q_{0}, p_{1}\right)=S_{c}\left(x_{0}, x_{1}\right)$, we obtain that the function

$$
x \longmapsto S_{c}\left(x_{-1}, x\right)+S_{c}\left(x, y_{1}\right)
$$

must have a minimum at $x_{0}$ and the equation

$$
\partial_{2} S_{c}\left(x_{-1}, x_{0}\right)=-\partial_{1} S_{c}\left(x_{0}, y_{1}\right)
$$

holds true. Let us take a given sequence $Q^{n}$ such that $q_{-1}^{n}$ has a limit $q_{-1}$, then any accumulation point of $p_{1}^{n}$ for any admissible sequence $P^{n}$ must have a covering that satisfies the above equation hence the sequence $p_{1}^{n}$ must have a limit, which corresponds to the only solution of the equation. We get that $p_{1}=q_{1}$ by applying this to the case $P^{n}=Q^{n}$.

The mapping

$$
\begin{gathered}
\Phi_{c}: \Sigma_{c} \longrightarrow \Sigma_{c} \\
q \longmapsto q_{1} .
\end{gathered}
$$

is Lipschitz continuous. We could prove it using arguments as in lemma 3 but more involved, we prefer to send the reader to [9]. The set

$$
\tilde{\Sigma}_{c}=\left\{\left(q, \partial_{1} S_{c}\left(q, \Phi_{c}(q)\right)\right), q \in \Sigma_{c}\right\}
$$

is $\Phi$-invariant. There is a map $\phi_{c}: \pi^{-1}\left(\Sigma_{c}\right) \longrightarrow \pi^{-1}\left(\Sigma_{c}\right)$ so that the following diagram commutes.

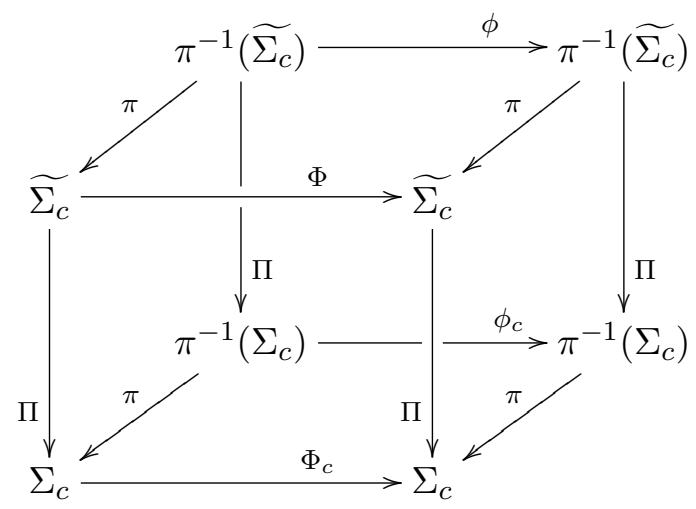


The vertical projections $\Pi$ on this diagram are all bilipschitz homeomorphisms. We will often use the notation $\Sigma_{c}$ for $\pi^{-1}\left(\Sigma_{c}\right)$ and $\tilde{\Sigma}_{c}$ for $\pi^{-1}\left(\tilde{\Sigma}_{c}\right)$. We have given the general definition of a Peierl's set. In order to obtain asymptotic orbits to it, some information must be obtained on its topology. We now focus our attention to the vicinity of the torus $\mathbb{T}^{n} \times\left\{y_{0}\right\}$. The following proposition provides us with some Peierl's set in this zone of phase space, and gives us the topological information we need.

Proposition 1 Let us fix $C>0$, there is a function $\delta(\epsilon)$ with $\lim _{\epsilon \longrightarrow 0} \delta(\epsilon)=0$, and a constant $A>0$ such that when $\epsilon$ is small enough we have

$$
\Sigma_{c} \subset U_{\delta(\epsilon)}
$$

and

$$
\tilde{\Sigma}_{c} \in\left\{\left\|y-y_{0}\right\| \leqslant A \sqrt{\epsilon}\right\} \in \mathbb{T}^{n} \times \mathbb{R}^{n}
$$

for any $c$ such that $\left\|c-\nabla h\left(\omega_{0}\right)\right\| \leqslant C \epsilon$.

In the following subsections, we prove proposition 1.

\subsection{Averaging}

Let us set

$$
\widetilde{S}\left(x_{0}, x_{1}\right)=S\left(x_{0}, x_{1}\right)+f\left(x_{0}\right)-f\left(x_{1}\right),
$$

where $f \in C^{1}\left(\mathbb{T}^{n}\right)$. All the functionals defined above on sets of periodic orbits are unchanged when $S$ is replaced by $\widetilde{S}$. We will use this fact to reduce our functionals to more convenient forms.

Lemma 4 For any $o>0$, There is a function $f_{o} \in C^{1}\left(\mathbb{T}^{n}\right)$, such that the modified generating function

$$
S_{o}\left(x_{0}, x_{1}\right)=S\left(x_{0}, x_{1}\right)+\epsilon f_{o}\left(x_{0}\right)-\epsilon f_{o}\left(x_{1}\right)
$$

satisfies

$$
S_{o}\left(x_{0}, x_{1}\right)=h\left(x_{1}-x_{0}\right)+\epsilon \mathcal{P}\left(x_{0}\right)+\epsilon R\left(x_{0}, x_{1}\right)
$$

with

$$
\left|R\left(x_{0}, x_{0}+\omega_{0}\right)\right| \leqslant o
$$

Proof: We first formally solve the equation

$$
P\left(x, x+\omega_{0}\right)+f(x)-f\left(x+\omega_{0}\right)=\mathcal{P}\left(x_{0}\right) .
$$

Let us put

$$
P\left(x, x+\omega_{0}\right)=\sum_{k \in \mathbb{Z}^{n}} p_{k} e^{i<k, x>}
$$

then

$$
\mathcal{P}(x)=\sum_{k \in R} p_{k} e^{i<k, x>}
$$

and the function $f(x)=\sum_{k \in \mathbb{Z}^{n}} f_{k} e^{i<k, x>}$, with

$$
f_{k}=\frac{p_{k}}{e^{i<k, \omega_{0}>}-1}, k \notin R ; f_{k}=0, k \in R
$$


formally solves the equation. Under our hypothesis, the above series can be divergent. Nevertheless, given $o>0$, there is $K \in \mathbb{R}$ such that

$$
\left|\sum_{|k| \geqslant K} p_{k} e^{i<k, x>}\right| \leqslant o .
$$

It is then easy to see that

$$
f_{o}(x)=\sum_{|k| \leqslant K} f_{k} e^{i<k, x>}
$$

satisfies

$$
\left\|P\left(x, x+\omega_{0}\right)+f_{o}(x)-f_{o}\left(x+\omega_{0}\right)-\mathcal{P}(x)\right\|_{\infty} \leqslant o .
$$

this proves lemma 4.

It will be useful in the following to use the notations

$$
\tilde{S}_{c}=S_{c}-\alpha(c), \tilde{H}_{c}=H_{c}-T \alpha(c),
$$

and

$$
\mathcal{Q}(x)=\mathcal{P}(x)-\min (\mathcal{P}) .
$$

Lemma 5 Let us fix $C>0$, there are non negative numbers $a$ and $b$ and a function $K(o)$ independent of $\epsilon$ such that for any $c \in \mathbb{R}^{n}$ satisfying $\left\|\nabla h\left(\omega_{0}\right)-c\right\| \leqslant C \epsilon$,

$$
a\|t\|^{2}+\epsilon \mathcal{Q}\left(x_{0}\right)-4 o \epsilon-K \epsilon^{2} \leqslant{ }_{c} \tilde{S}_{o}\left(x_{0}, x_{1}\right) \leqslant b\|t\|^{2}+\epsilon \mathcal{Q}\left(x_{0}\right)+4 o \epsilon+K \epsilon^{2},
$$

where $t=x_{1}-x_{0}-\omega_{0}$.

Proof: For $\epsilon$ small enough, the function $x_{1} \longmapsto h\left(x_{1}-x_{0}\right)+\epsilon R\left(x_{0}, x_{1}\right)$ is uniformly strictly convex thus there exists $a>0$ such that

$$
\begin{gathered}
h\left(x_{1}-x_{0}\right)+\epsilon R\left(x_{0}, x_{1}\right) \geqslant \\
h\left(\omega_{0}\right)+\epsilon R\left(x_{0}, x_{0}+\omega_{0}\right)+<\nabla h\left(\omega_{0}\right)+\epsilon \partial_{2} R\left(x_{0}, x_{0}+\omega_{0}\right), x_{1}-x_{0}-\omega_{0}>+a\left\|x_{1}-x_{0}-\omega_{0}\right\|^{2} .
\end{gathered}
$$

This gives

$$
\begin{aligned}
{ }_{c} S_{o}\left(x_{0}, x_{1}\right) \geqslant & h\left(\omega_{0}\right)-<c, \omega_{0}>-\epsilon O+<\nabla h\left(\omega_{0}\right)+\epsilon \partial_{2} R\left(x_{0}, x_{0}+\omega_{0}\right)-c, t> \\
& +a\|t\|^{2}+\epsilon \mathcal{P}\left(x_{0}\right) \\
\geqslant & h\left(\omega_{0}\right)-<c, \omega_{0}>-\epsilon O-\epsilon C\|t\|+a\|t\|^{2}+\epsilon \mathcal{P}\left(x_{0}\right) \\
\geqslant & h\left(\omega_{0}\right)-<c, \omega_{0}>-\epsilon O-K \epsilon^{2}+a\|t\|^{2}+\epsilon \mathcal{P}\left(x_{0}\right)
\end{aligned}
$$

changing the constant $a$ in the last inequation. We can also obtain the majoration

$$
{ }_{c} S_{o}\left(x_{0}, x_{1}\right) \leqslant h\left(\omega_{0}\right)-<c, \omega_{0}>+\epsilon O+K \epsilon^{2}+b\|t\|^{2}+\epsilon \mathcal{P}\left(x_{0}\right) .
$$

We are now in a position to estimate $\alpha(c)$. The above minoration gives

$$
\begin{aligned}
\alpha(c) & \geqslant h\left(\omega_{0}\right)-<c, \omega_{0}>+\epsilon \min \mathcal{P}-\epsilon O-K \epsilon^{2} \\
& \geqslant h\left(\omega_{0}\right)-<c, \omega_{0}>+\epsilon \min \mathcal{P}-2 \epsilon O
\end{aligned}
$$


for $\epsilon$ small enough with respect to $o$. Let $x$ and $y$ be two points of $\Gamma_{0}$, consider the configuration $x_{k}=x+k \omega_{0}$, let $T$ be the time between $\epsilon^{-1 / 2}$ and $2 \epsilon^{-1 / 2}$ for which $x_{T}$ is closest to $y$ and set $d=y-x_{T}$. Now consider the configuration $Y=\left(y_{k}=x_{k}+k d / T\right)$, it connects $x$ and $y$ and satisfies

$$
{ }_{c} L_{o}(Y) \leqslant b T^{-1}\|d\|^{2}+T \epsilon \min (\mathcal{P})+T h\left(\omega_{0}\right)-T<c, \omega_{0}>+\epsilon o T+\epsilon^{2} K T
$$

because

$$
{ }_{c} S_{o}\left(y_{k}, y_{k+1}\right) \leqslant b\|d / T\|^{2}+\epsilon \mathcal{P}\left(y_{k}\right)+h\left(\omega_{0}\right)-<c, \omega_{0}>+o \epsilon+K \epsilon^{2}+h\left(\omega_{0}\right) .
$$

Since $d$ goes to zero as $\epsilon$ goes to zero, we obtain for $\epsilon$ small enough the estimate

$$
{ }_{c} L_{o}(Y) \leqslant T \epsilon \min (\mathcal{P})+T h\left(\omega_{0}\right)+T<c, \omega_{0}>+2 o \sqrt{\epsilon}
$$

If we apply this remark to the case $x=y, Y$ is a periodic configuration and we get

$$
\alpha(c) \leqslant h\left(\omega_{0}\right)-<c, \omega_{0}>+\epsilon \min \mathcal{P}+2 o \epsilon .
$$

We have estimated $\alpha(c)$ :

$$
\left|\alpha(c)-h\left(\omega_{0}\right)+<c, \omega_{0}>-\epsilon \min \mathcal{P}\right| \leqslant 2 o \epsilon .
$$

The inequality (3) is obtained by mixing this estimate with the above inequalities. We have also proved:

Lemma 6 Let $x$ and $y$ be two points of $\Gamma_{0}$, there is a configuration $X=\left(x=x_{0}, \ldots, x_{T}=y\right)$ with $\epsilon^{-1 / 2} \leqslant T \leqslant 2 \epsilon^{-1 / 2}$ and

$$
{ }_{c} \tilde{L}_{o}(X) \leqslant 10 o \sqrt{\epsilon} \text {. }
$$

\subsection{Localization of the asymptotic set}

In this rather technical section, we prove proposition 1. In the following calculations, we will mainly use inequality (3), thus our result will be true for any $c$ satisfying the hypothesis of lemma 5. We will omit the subscript $c$.

Lemma 7 Let $\nu>0$ and $x$ and $y$ be two points of $U_{\nu}$, for $o>0$ small enough and $\epsilon$ small enough with respect to $\alpha$, there is a configuration $X=\left(x=x_{0}, \ldots, x_{T}=y\right)$ satisfying

$$
\tilde{L}_{o}(X) \leqslant C \nu^{2} \sqrt{\epsilon} .
$$

Proof: We will construct our connecting configuration via $\Gamma_{0}$. Let $x \in U_{\nu}, x+d$ its projection on $\Gamma_{0}$. Considering the configuration $\left(x_{0}, \ldots, x_{T}\right)=\left(x, x+\omega_{0}+d / T, \ldots, x+T \omega_{0}+d\right)$, we get

$$
\tilde{S}_{o}\left(x_{i}, x_{i+1}\right) \leqslant b\|d / T\|^{2}+\epsilon \mathcal{Q}\left(x+i\left(\omega_{0}+d / T\right)\right)+\epsilon O+K \epsilon^{2} .
$$

Adding these inequalities gives

$$
\tilde{L}_{o}(X) \leqslant b \nu^{2} / T+T \epsilon\left(\sup _{U_{\nu}} \mathcal{Q}+o+K \epsilon\right),
$$


and since

$$
\begin{gathered}
\mathcal{P}(x) \leqslant \min \mathcal{P}+C\left(d\left(x, \Gamma_{0}\right)\right)^{2}, \\
\tilde{L}(X) \leqslant b \nu^{2} / T+T \epsilon\left(C \nu^{2}+4 o+K \epsilon\right) .
\end{gathered}
$$

Taking $T=\sqrt{\epsilon}$ we obtain that

$$
\tilde{L}_{\alpha}\left(x_{0}, \ldots, x_{T}\right) \leqslant C \nu^{2} \sqrt{\epsilon} .
$$

In order to prove the lemma, we just have to consider a three part configuration

$$
x=x_{0}, \ldots, x_{N}, \ldots, x_{M}, \ldots, x_{T}=y,
$$

where $x_{N} \in \Gamma_{0}$ and $x_{0}, \ldots, x_{N}$ is the orbit considered above, $x_{M} \in \Gamma_{0}$ and $x_{M}, \ldots, x_{T}$ is a reversed configuration like above, and $x_{N}, \ldots, x_{M}$ is a configuration obtained by lemma 6 . The inequality of the lemma is then satisfied if $o$ is small enough.

Corollary 1 Let $x$ and $y$ lie in $U_{\nu}$, any configuration connecting $x$ and $y$ satisfies

$$
\tilde{L}_{\alpha} \geqslant-C \nu^{2} \sqrt{\epsilon}
$$

Proof: Let us consider a configuration connecting $x$ and $y$, and complete it by the configuration of low action obtained above connecting $y$ and $x$. We obtain a periodic configuration, writing that its action must be non negative gives the inequality of the corollary.

Let $0<\nu<\delta$, and let $W=\mathbb{R}^{n}-U_{\nu}$.

Lemma 8 Let $X=x_{0}, \ldots, x_{T}$ be a configuration with $x_{0} \in U_{\nu}, x_{i} \in W$ for $i \geqslant 1$ and $x_{T} \in \mathbb{R}^{n}-U_{\delta}$. Then for $\alpha$ and $\epsilon$ small enough

$$
\tilde{L}_{\alpha}(X) \geqslant D \sqrt{\epsilon} \nu(\delta-\nu) .
$$

The conclusion holds true for a configuration with $x_{T} \in U_{\nu}, x_{i} \in W$ for $i<T$ and $x_{0} \in$ $\mathbb{R}^{n}-U_{\delta}$.

Proof: We will give the proof of the first part of the lemma, the proof of the second part goes along the same line and is easier. Since $\Gamma_{0}$ is a nondegenerate minimal manifold for $\mathcal{P}$ we have

$$
\mathcal{P}(x) \geqslant \min \mathcal{P}+D\left(d\left(x, \Gamma_{0}\right)\right)^{2} .
$$

It follows that

$$
\tilde{S}_{o}\left(x_{i}, x_{i+1}\right) \geqslant a\left\|t_{i}\right\|^{2}+\epsilon C \nu^{2}-4 o \epsilon-K \epsilon^{2}
$$

for $i \geqslant 1$. We then choose $o$ so that $D \nu^{2}-4 o \geqslant D \nu^{2} / 2>0$, and obtain

$$
\tilde{S}_{o}\left(x_{i}, x_{i+1}\right) \geqslant C\left\|t_{i}\right\| \nu \sqrt{\epsilon},
$$

for $\epsilon$ small enough and $i \geqslant 1$. On the other hand,

$$
\begin{gathered}
\tilde{S}_{o}\left(x_{0}, x_{1}\right) \geqslant a\left\|t_{0}\right\|^{2}+\epsilon \mathcal{Q}\left(x_{0}\right)-4 o \epsilon-K \epsilon^{2}, \\
\tilde{S}_{o}\left(x_{0}, x_{1}\right) \geqslant a\left\|t_{0}\right\|^{2}+\epsilon D \nu^{2}-\epsilon C\left\|t_{0}\right\|-4 o \epsilon-K \epsilon^{2} .
\end{gathered}
$$

For $\epsilon$ small enough, we get

$$
\tilde{S}_{o}\left(x_{0}, x_{1}\right) \geqslant D\left\|t_{0}\right\| \nu \sqrt{\epsilon}
$$


Adding all the minorations we have obtained gives the lemma because

$$
\sum_{i}\left\|t_{i}\right\| \geqslant\left\|\sum_{i} t_{i}\right\| \geqslant|\delta-\nu| .
$$

Lemma 9 There is a function $\delta(\epsilon)$ such that $\lim _{\epsilon \rightarrow 0} \delta(\epsilon)=0$ and a function a $(\epsilon)$ satisfying $a(\epsilon)>0$ for $\epsilon>0$ such that any periodic configuration $\left(x_{0}, x_{1}, \ldots x_{0}+m\right)$ of any rotation number leaving $U_{\delta(\epsilon)}$, satisfies $L\left(x_{0}, \ldots, x_{0}+m\right) \geqslant 2 a(\epsilon)$ :

$$
L\left(x_{0}, \ldots, x_{0}+m\right) \geqslant 2 a(\epsilon) \text { if } x_{i} \notin U_{\delta(\epsilon)} \text { for some } i .
$$

Proof: Let $\delta$ be fixed, and let $X$ be a periodic configuration leaving $U_{\delta}$, let $\nu=\delta^{2}$. If $X$ stays out of $U_{\nu}$, then adding the inequalities

$$
\tilde{S}_{o}\left(x_{i}, x_{i+1}\right) \geqslant a\left\|t_{i}\right\|^{2}+\epsilon D \nu^{2}-4 o \epsilon-K \epsilon^{2},
$$

we obtain

$$
\tilde{L}(X)=\tilde{L}_{o}(X) \geqslant \epsilon D \nu^{2}-4 o \epsilon-K \epsilon^{2},
$$

for any small $o$, so that we have

$$
\tilde{L}(X) \geqslant D \epsilon \delta^{4}
$$

for $\epsilon$ small enough. Let us now suppose that $X$ comes into $U_{\nu}$. We can find a configuration $Y=x_{-i}, \ldots, x_{0}, \ldots, x_{j}$ in $X$ with $x_{0} \notin U_{\delta}, x_{k} \in W$ if $-i<k<j$ and $\left(x_{-i}, x_{j}\right) \in\left(U_{\nu}\right)^{2}$. The above lemma gives us

$$
\tilde{L}_{\alpha}(Y) \geqslant D \sqrt{\epsilon} \delta^{3}
$$

By corollary 3, we then have

$$
\tilde{L}(X) \geqslant \sqrt{\epsilon}\left(D \delta^{3}-C \delta^{4}\right)
$$

when $\epsilon$ is small enough with respect to delta. As a consequence of these results, there is a constant $D>0$ such that the inequality

$$
\tilde{L}(X) \geqslant D \min \left(\sqrt{\epsilon} \delta^{3}, \epsilon \delta^{4}\right)
$$

must be satisfied when $\delta$ is small enough and when $\epsilon$ is small enough with respect to $\delta$. The lemma follows.

The horizontal localization of proposition 1 follows. Let us now end the proof of proposition 1 : We are still given a fixed $c$ satisfying the hypothesis of theorem 1 , and we omit all subscripts $c$. Let $X^{k} \in E_{w_{k}}^{T_{k}}$ be a sequence of periodic configurations such that $\tilde{L}\left(X^{k}\right) \longrightarrow 0$ and $T_{k} \longrightarrow \infty$.

Lemma 10 The rotation number $w_{k}$ satisfies:

$$
\left\|w_{k} / T_{k}-\omega_{0}\right\| \leqslant 2 A \sqrt{\epsilon}
$$

when $k$ large enough and $\epsilon$ small enough, where $A$ is the constant of inequality (2). 
Proof: By inequality (2), we have

$$
\left\|x_{i+1}^{k}-x_{i}^{k}-w_{k} / T_{k}\right\| \leqslant A \sqrt{\epsilon} .
$$

Assume that $\left\|w_{k} / T_{k}-\omega_{0}\right\| \geqslant 2 A \sqrt{\epsilon}$ with the same constant $A$, then setting $t_{i}^{k}=x_{i+1}^{k}-x_{i}^{k}-\omega_{0}$ as usual, we have that $\left\|t_{i}^{k}\right\| \geqslant A \sqrt{\epsilon}$. Putting this in inequality 3 gives

$$
\tilde{L}\left(X^{k}\right) \geqslant T_{k}\left(a A \epsilon-o \epsilon-K \epsilon^{2}\right)
$$

for any $o$, which gives a contradiction when $o$ and $\epsilon$ are small enough because $T_{k} \longrightarrow \infty$ and $L\left(X^{k}\right) \longrightarrow 0$.

Using lemma 10 and (2) we obtain

$$
\left\|x_{i+1}^{k}-x_{i}^{k}-\omega_{0}\right\| \leqslant 3 A \sqrt{\epsilon}
$$

for $k$ large enough. Vertical localization of proposition 1 follows, with a different constant $A$.

\section{Convergence}

In this section we will study sequences of periodic orbits, and obtain homoclinic orbits as accumulation points. We fix $c \in B_{C \epsilon}\left(\nabla\left(\omega_{0}\right)\right)$, and we will omit the subscript $c$ in the following Let $Q^{k}$ be a sequence of $T_{k}$-periodic orbits such that $\tilde{H}\left(Q^{k}\right) \longrightarrow 0$ and $T_{k} \longrightarrow \infty$. The orbit $Q^{k}$ has a covering $X^{k} \in E_{w_{k}}^{T_{k}}$ minimizing $L_{w_{k}}^{T_{k}}$. We can take a subsequence to be reduced to the case where the inequality of lemma 10 is satisfied for every $k$, and where the sequence $w_{k} / T_{k}$ has a limit $\omega$, which satisfies

$$
\left\|\omega-\omega_{0}\right\| \leqslant 2 A \sqrt{\epsilon} .
$$

Let $X^{k}(m) \in E_{w_{k}+m}^{T_{k}}$ be the minimizing sequence of $L_{w_{k}+m}^{T_{k}}$, and $Q^{k}(m)$ its associated orbit on $\mathbb{T}^{n}$. We need some informations about these orbits.

Lemma 11 There is a constant $C(m)$, depending on $m$ but not on $k$, such that

$$
\tilde{L}\left(X^{k}(m)\right) \leqslant C(m) .
$$

Proof:

$$
\begin{aligned}
\tilde{L}\left(X^{k}(m)\right) & \leqslant \tilde{L}\left(x_{0}^{k}, \ldots, x_{T_{k}-1}^{k}, x_{T_{k}}^{k}+m\right) \\
& \leqslant \tilde{L}\left(X^{k}\right)+\tilde{S}\left(x_{T_{k}-1}^{k}, x_{T_{k}}^{k}+m\right)-\tilde{S}\left(x_{T_{k}-1}^{k}, x_{T_{k}}^{k}\right) \\
& \leqslant \tilde{L}\left(X^{k}\right)-\min \tilde{S}+\sup _{\|x\| \leqslant 1,\|y\| \leqslant 1+m+\omega_{0}+3 A \sqrt{\epsilon}} \tilde{S}(x, y) .
\end{aligned}
$$

Lemma 12 There is a constant $C(m)$ such that for any $0 \leqslant i<j \leqslant T_{k}$

$$
\tilde{H}\left(q_{i}^{k}(m), \ldots, q_{j}^{k}(m)\right) \leqslant C(m) .
$$


Proof: Let us set $P=\left(p_{j}, \ldots, p_{i}\right)=\left(q_{j}^{k}(m), \ldots, q_{T_{k}}^{k}(m), q_{1}^{k}(m), \ldots, q_{i}^{k}(m)\right)$. Writing

$$
\tilde{H}(P)+\tilde{H}\left(q_{i}^{k}(m), q_{j}^{k}(m)\right) \geqslant 0
$$

gives

$$
\tilde{H}(P) \geqslant-\max _{\left(\mathbb{T}^{n}\right)^{2}} \tilde{H}
$$

We get

$$
\begin{aligned}
\tilde{H}\left(q_{i}^{k}(m), \ldots, q_{j}^{k}(m)\right) & =\tilde{H}\left(Q^{k}(m)\right)-\tilde{H}(P) \\
& \leqslant C(m)+\max \tilde{H} .
\end{aligned}
$$

Thanks to these lemmas, we are in a position to study the accumulation points of the sequence $Q^{k}(m)$. Let us fix a sequence $a_{k}$ of integers, we can consider the centered orbit

$$
\Theta^{k}=\left(\theta_{i}^{k}=q_{i+a_{k}}^{k}(m)\right)_{i \in \mathbb{Z}}
$$

where everything is defined by periodic continuation. There is a subsequence $\Theta^{l(k)}$ such that $l \longmapsto \theta_{i}^{l}$ has a limit $\theta_{i}$ for every $i$. The biinfinite sequence $\Theta=\left(\theta_{i}\right)_{i \in \mathbb{Z}}$ will be called an accumulation point of the centered orbit $\Theta^{k}$. An accumulation point of a centered orbit of a sequence $Y^{k}$ of periodic orbits will be called an accumulation point of $Y^{k}$. We have the following proposition, in which as usual everything depends on a vector $c \in \mathbb{R}^{n}$ satisfying $\left\|c-\nabla h\left(\omega_{0}\right)\right\| \leqslant C \epsilon$.

Proposition 2 Any accumulation point of $Q^{k}(m)$ is the projection of an orbit that is homoclinic to $\tilde{\Sigma}$, any orbit obtained that way satisfies the localization $\left\|y-y_{0}\right\| \leqslant A \sqrt{\epsilon}$ of theorem 1.

This proposition provides no existence result up to now since the accumulation point could be an orbit contained in $\Sigma$.

Proof: Since $\Theta^{k}$ are orbit segments, we have

$$
\partial_{2} S\left(\theta_{i-1}^{k}, \theta_{i}^{k}\right)=-\partial_{1} S\left(\theta_{i}^{k}, \theta_{i+1}^{k}\right),
$$

taking the limit we obtain

$$
\partial_{2} S\left(\theta_{i-1}, \theta_{i}\right)=-\partial_{1} S\left(\theta_{i}, \theta_{i+1}\right),
$$

which means that $\theta_{i}$ is the projection of the orbit $\left(\theta_{i}, \partial_{1} S\left(\theta_{i}, \theta_{i+1}\right)\right)$. Since $\| x_{i+1}^{k}(m)-x_{i}^{k}(m)-$ $\omega_{0} \| \leqslant 4 A \sqrt{\epsilon}$, this orbit must stay in the zone $\left\|y-y_{0}\right\| \leqslant A \sqrt{\epsilon}$ (where $\mathrm{A}$ is a new constant). To prove that this orbit is homoclinic, it is enough to prove that the $\alpha$ and $\omega$-limit of the orbit $\left(\theta_{i}, \partial_{1} S\left(\theta_{i}, \theta_{i+1}\right)\right)$ lie in $\tilde{\Sigma}$. We will prove it for the $\omega$-limit, the other part being similar. It is sufficient to see that for any subsequence $\theta_{j(i)}$ having a limit $\theta$, we have $\theta \in \Sigma_{c}$ and $\theta_{j+1} \longrightarrow \phi_{c}(\theta)$. Taking a subsequence if necessary, we can suppose that $d\left(\theta_{j}, \theta\right) \leqslant 2^{-j}$. Let us consider the configuration

$$
Z_{j}=\left(\theta, \theta_{j(i)+1}, \ldots, \theta_{j(i+1)-1}, \theta\right)
$$


it satisfies $\tilde{H}\left(Z_{j}\right) \geqslant 0$ and

$$
\tilde{H}\left(Z_{j}\right) \leqslant \tilde{H}\left(\theta_{j(i)}, \theta_{j(i)+1}, \ldots, \theta_{j(i+1)}\right)+C 2^{-j} .
$$

Adding these inequalities and using lemma 11 yields

$$
\begin{aligned}
\sum_{i=i_{0}}^{i_{1}} \tilde{H}\left(Z_{j(i)}\right) & \leqslant C 2^{-j\left(i_{0}\right)}+\tilde{H}\left(\theta_{j\left(i_{0}\right)}, \ldots, \theta_{j\left(i_{1}+1\right)}\right) \\
& \leqslant C 2^{-j\left(i_{0}\right)}+\sup _{k}\left(\tilde{H}\left(\theta_{j\left(i_{0}\right)}^{k}, \ldots, \theta_{j\left(i_{1}+1\right)}^{k}\right)\right) \\
& \leqslant C 2^{-j\left(i_{0}\right)}+C(m) .
\end{aligned}
$$

We obtain that $\tilde{H}\left(Z_{i}\right) \longrightarrow 0$ so that $\theta \in \Sigma_{c}$ and $\theta_{j(i)+1} \longrightarrow \phi_{c}(\theta)$. The last assertion follows from the fact that $\phi_{c}(\theta)$ is the only possible accumulation point of the sequence $\theta_{j(i)+1}$.

\section{$5 \quad$ Nontriviality and multiplicity}

As we noticed it in the previous section, proposition 2 does not guarantee the existence of nontrivial homoclinic orbits. Some additional work will be needed. Let us fix $\delta$ small, there is $\epsilon_{0}$ such that $\Sigma \in U_{\delta}$ when $\epsilon \leqslant \epsilon_{0}$. There also exists $\eta$ such that $d(q, \Sigma) \leqslant \eta \Rightarrow q \in U_{\delta}$ when $\epsilon \leqslant \epsilon_{0}$. Let us define the open set

$$
V_{\eta} \subset \mathbb{R}^{n} \times \mathbb{R}^{n}=\left\{\left(x_{0}, x_{1}\right) \text { such that } d\left(\left(x_{0}, x_{1}\right), \operatorname{graph}\left(\phi_{c}\right)\right)<\eta\right\} \subset U_{\delta} \times U_{\delta} .
$$

We associate to any periodic orbit $X \in E_{w}^{T}$ its relative homology

$$
\left(h_{b}(X)=<w, b>-T<\omega_{0}, b>\right)_{b \in B} \in \mathbb{Z}^{r},
$$

recall that $B$ is a basis of the resonant group. We also associate to any point $x \in U_{\delta}$ coefficients

$$
\left(h_{b}(x)\right)=E(<x, b>)_{b \in B} \in \mathbb{Z}^{r},
$$

where $E(x)$ is the integer closest to $x$, the above giving a good definition if $\delta$ has been chosen small enough. These coefficients have of course no topological meaning.

Lemma 13 If $\delta$ has been chosen small enough, for any point $x \in \Sigma$, we have

$$
h_{b}\left(\phi_{c}(x)\right)=h_{b}(x)+<\omega_{0}, b>
$$

from which easily follows that

$$
\left(x_{0}, x_{1}\right) \in V_{\eta} \Rightarrow h_{b}\left(x_{0}\right)=h_{b}\left(x_{1}\right)+<\omega_{0}, b>.
$$

Proof: Let us consider a sequence $X^{k}$ as above with $\tilde{L}\left(X^{k}\right) \longrightarrow 0$ and $x_{0}^{k} \longrightarrow x$. The sequence $x_{1}^{k}$ then has a limit $\phi_{c}(x)$ satisfying $\left\|\phi_{c}(x)-x-\omega_{0}\right\| \leqslant A \sqrt{\epsilon}$. We obtain that $\mid<\phi_{c}(x), b>-\left\langle x, b>-<\omega_{0}, b>\right|$ is small. Since both $\phi_{c}(x)$ and $x$ are in $U_{\delta}$, $\left.<\phi_{c}(x), b\right\rangle$ and $\langle x, b\rangle$ are close to integers, the result follows. 
As an easy corollary of that lemma, we obtain that if $X^{k}$ is an orbit such that $\tilde{L}\left(X^{k}\right) \longrightarrow 0$ then $\left(x_{i}^{k}, x_{i+1}^{k}\right) \in V_{\eta}$ for all $i$ when $k$ large enough, thus $h_{b}\left(X^{k}\right)=<x_{T_{k}}^{k}, b>-<x_{0}^{k}, b>$ $-T_{k}<\omega_{0}, b>$ is close to $h_{b}\left(x_{T_{k}}^{k}\right)-h_{b}\left(x_{0}^{k}\right)-T_{k}<\omega_{0}, b>=0$, so $h_{b}\left(X^{k}\right)=0$, which writes

$$
<w_{k}, b>=<\omega_{0}, b>
$$

for $k$ large enough. We also define the relative homology of a $\phi$-orbit homoclinic to $\Sigma$ as follows,

$$
h_{b}(\Theta)=\lim _{i \longrightarrow \infty}\left(h_{b}\left(\theta_{i}\right)-h_{b}\left(\theta_{-i}\right)-2 i<\omega_{0}, b>\right) .
$$

This is well defined because for $i$ large enough, we have $\theta_{i} \in U_{\delta}$ and $\left(\theta_{i}, \theta_{i+1}\right) \in V_{\eta}$. We can now prove the existence of nontrivial orbits. Let us fix some $m$ and consider the sequence $X^{k}(m)$. There is an alternative

1- There exist $a_{k}$ such that $\left(x_{a_{k}}^{k}(m), x_{a_{k}+1}^{k}(m)\right)$ admits an accumulation point out of $V_{\eta}$, and so there is a nontrivial homoclinic, that leaves $U_{\eta}$.

2- $\left(x_{i}^{k}(m), x_{i+1}^{k}(m)\right) \in V_{\eta}$ holds when $k$ is large enough.

If 2- holds, it follows easily that the periodic orbit $X^{k}(m)$ has trivial relative homology:

$$
h_{b}\left(X^{k}(m)\right)=0 .
$$

This last equation writes

$$
<m, b>=0,
$$

we can choose $m$ so that this equation is not satisfied, in this case 2- is impossible and so 1is true.

We will now go in some kind of concentration compactness for our sequences $X^{k}(m)$ to obtain multiplicity. Assume $m$ has been chosen so that a nontrivial accumulation orbit leaving $U_{\eta}$ exists. There are a sequence $a_{0}^{k}$, an homoclinic orbit $\Theta^{0}$ and a subsequence of $X^{k}(m)$ that will still be noted $X^{k}(m)$ such that $x_{i+a_{0}^{k}}^{k}(m) \longrightarrow \theta_{i}^{0}$ for all $i$. Possibly changing $a_{0}^{k}$, we can suppose that there exists $A^{0}$ such that $\left(\theta_{i}^{0}, \theta_{i+1}^{0}\right) \in V_{\eta}$ for $i<0$ or $i \geqslant A^{0}$, and given $\nu>0$, we can take a subsequence of our subsequence such that $d\left(x_{i+a_{0}^{k}}^{k}(m), \theta_{i}^{0}\right) \leqslant \nu$ for $|i| \leqslant 2 A^{0}$, this subsequence is still noted $X^{k}(m)$. Let us define

$$
a_{1}^{k}=\min \left\{i \geqslant a_{0}^{k}+A^{0} \text { such that }\left(x_{i}^{k}(m), x_{i+1}^{k}(m)\right) \notin V_{\eta}\right\}
$$

and

$$
a_{-1}^{k}=\max \left\{i<a_{0}^{k} \text { such that }\left(x_{i}^{k}(m), x_{i+1}^{k}(m)\right) \notin V_{\eta}\right\} .
$$

We can extract another subsequence such that there exists a nontrivial homoclinic orbit $\Theta^{1}$, and an integer $A^{1}$ satisfying

$$
\left(\theta_{i}^{1}, \theta_{i+1}^{1}\right) \in V_{\eta} \text { when } i \geqslant A^{1}
$$

and

$$
d\left(x_{i+a_{1}^{k}}^{k}(m), \theta_{i}^{1}\right) \leqslant \nu \text { when }|i| \leqslant 2 A^{1} .
$$

We can continue the process by defining

$$
a_{l}^{k}=\min \left\{i \geqslant a_{l-1}^{k}+A^{l-1} \text { such that }\left(x_{i}^{k}(m), x_{i+1}^{k}(m)\right) \notin V_{\eta}\right\}
$$


and finding $\Theta^{l}$ and $A^{l}$ with

$$
\left(\theta_{i}^{l}, \theta_{i+1}^{l}\right) \in V_{\eta} \text { when } i \geqslant A^{l}
$$

and

$$
d\left(x_{i+a_{l}^{k}}^{k}(m), \theta_{i}^{l}\right) \leqslant \nu \text { when }|i| \leqslant 2 A^{l} .
$$

We can do the same for $l<0$. Assume now that the process ends on each side, that is we have $l^{+} \geqslant 0$ and $l^{-} \leqslant 0$ such that $a_{l^{++1}}^{k} \geqslant T_{k}$ and $a_{l^{-}-1}^{k}<0$ when $k \geqslant k_{0}$, then we have found a subsequence $X^{k}(m)$ such that

$$
d\left(x_{i}^{k}(m), \theta_{i-a_{l}^{k}}^{l}\right) \leqslant \nu \text { when } a_{l}^{k}-2 A^{l} \leqslant i \leqslant a_{l}^{k}+2 A^{l}
$$

and

$$
\left(x_{i}^{k}(m), x_{i+1}^{k}(m)\right) \in V_{\eta} \text { when } a_{l}^{k}+A^{l} \leqslant i<a_{l+1}^{k} \text { or } T_{k}>i \geqslant a_{l^{+}}^{k}+A^{l^{+}} \text {or } 0 \leqslant i<a_{l^{-}}^{k} .
$$

Looking precisely at the construction we just performed and applying lemma 13, we see that

$$
<m, b>=h_{b}\left(X^{k}(m)\right)=\sum_{l=l^{-}}^{l^{+}} h_{b}\left(\Theta^{l}\right) .
$$

Lemma 14 If there are only a finite number $N$ of geometrically distinct homoclinics then the process described above ends for each $m$. It follows that the relative homologies of these homoclinics must additively generate $\mathbb{Z}^{r}$ and thus

$$
N \geqslant r+1 \text {. }
$$

Proof: If the process always ends, then the above sum formula (4) must be satisfied, and since the function

$$
m \longmapsto(<m, b>)_{b \in B} \in \mathbb{Z}^{r}
$$

is easily seen to be onto, we have the second part of the lemma. It remains to prove that the process ends. If there are only $N$ homoclinic orbits, there are at most $N$ leaving $U_{\eta}$, one of them must occur at least $\left(l^{+}-l^{-}\right) / N$ times in the sequence $\Theta^{l}$ defined above, that is there are indices $l_{1}, \ldots, l_{K}$ with $K \geqslant\left(l^{+}-l^{-}\right) / N$ such that $\Theta^{l_{j}}=\Theta$. Let us set

$$
Y_{j}^{k}=\left(x_{a_{l_{j}}^{k}}^{k}, x_{a_{l_{j}}^{k}+1}^{k}, \ldots, x_{a_{l_{j+1}}^{k}}^{k}\right) \text {. }
$$

and

$$
\tilde{Y}_{j}^{k}=\left(\theta_{0}, x_{a_{l_{j}}^{k}+1}^{k}, \ldots, x_{a_{l_{j+1}}^{k}-1}^{k}, \theta_{0}\right) .
$$

The configuration $\tilde{Y}_{j}^{k}$ is periodic and leaves $U_{\eta}$, so there must be $o>0$ such that

$$
\tilde{L}\left(\tilde{Y}_{j}^{k}\right) \geqslant 2 o .
$$

If $\nu$ has been chosen small enough, it follows that

$$
\tilde{L}\left(Y_{j}^{k}\right) \geqslant o .
$$

This gives

$$
C(m) \geqslant L\left(X^{k}(m)\right) \geqslant K o
$$

which is a majoration for $K$ and thus for $l^{+}-l^{-}$.

Acknowledgments : I thank Professor E. Séré for his comments and encouragement. I thank the referee for useful remarks. 


\section{References}

[1] Arnold V. I. : Instability of Dynamical systems with several degrees of freedom, Soviet Math. 5 (1964), 581-585.

[2] Bernstein D. - Katok A. : Birkhoff periodic orbits for small perturbations of completely integrable Hamiltonian systems with convex Hamiltonian, Invent. Math. 88(1987), 227241.

[3] Bolotin S.V. : Homoclinic orbits to invariant tori of Hamiltonian systems, A. M. S. Translations, ser. $2, \mathbf{1 6 8}$ (1995), 21-90.

[4] Mc Duff D. - Salamon D. : Introduction to symplectic topology, Oxford Math. Monographs (1995).

[5] L. H. Eliasson : Biasymptotic Solutions of Perturbed Integrable Hamiltonian Systems, Bol. Soc. Brasil Mat. (N.S.) 25 (1994), 56-76.

[6] Fathi A. : Orbites hétéroclines et ensembles de Peierls, CRAS 326 (1998), 1213-1216.

[7] Lochak P. : Canonical perturbation theory via simultaneous approximation, Russ. Math. Surveys 47 (1992) 57-133.

[8] Lochak P. : Arnold's diffusion : a compendium of remarks and questions, in Proceedings of the Barcelona 1995 conference on dynamical systems with more than 3 degrees of freedom, Kluwer, (1996).

[9] Mather J. : Action minimizing invariant measures for positive definite Lagrangian systems, Math. Z. 207 (1991), 169-207.

[10] Mather J. :Variational construction of connecting orbits, Ann. Inst. Fourier, 43 (1993), $1349-1368$.

[11] Treschev D. : The mechanism of destruction of resonant tori of Hamiltonian systems, Math Sb, (1989), in English in Math. USSR Sbornik 68 (1991), 181-203.

Patrick Bernard

Institut Fourier,

Université Grenoble I, BP 74, 38402 Saint Martin d'Hères cedex, France.

Patrick.Bernard@ujf-grenoble.fr 